Editorial

\title{
The Use of Mobile Technology in Audiology: Transforming Clinical Practice with Smartphones
}

\author{
Devin L. McCaslin ${ }^{1}$ Deputy Editor-in-Chief \\ ${ }^{1}$ Department of Audiology, Mayo Clinic, Rochester, Minnesota
}

J Am Acad Audiol 2020;31:699-700.

There is no question that smartphones have become an integral part of our lives. I am a Generation Xer and, during my childhood, phones were used to simply make phone calls. In fact, my family had what was referred to as a party line. This was a phone line that was shared by several households and, if someone picked up the phone at your house while the line was in use by another house, you would join their conversation.

In the 1980s, home computers became more and more commonplace and televisions became larger. During this time, the internet was available in large institutions such as universities and, by the 90 s, was becoming accessible to those at home. Fast forward to today and all of these technologies have merged into what has been termed mobile technology. We now have a television, phone, and computer in our pocket, all connected to the internet.

With the recent introduction of $5 \mathrm{G}$ mobile networks, we will be able to watch our favorite shows or sports anywhere without interruption. In the United States, approximately 257 million people own smartphones and this is expected to grow to 290 million in 2024. The majority of our time on these phones (90 percent) is spent using software applications (apps), rather than making a telephone call.

According to Statista, a statistics portal, there are upward of 6.3 million apps. These mobile apps cover the areas of social media, games, shopping, banking, and much more. One area that is seeing explosive growth is the use of mobile apps to connect patients to their health-care providers. Mobile health-care technology is currently being used in areas such as cardiology (mobile EKG), dermatology, and diabetes, to name just a few.

Audiology is also leveraging mobile technology to change how we connect to, and manage, our patients and their care. We are using mobile technologies to screen hearing-and boothless audiometry is quickly becoming a reality. In the area of cochlear implants, we can track electrode impedances in real time and adjust the cochlear implant programs remotely using mobile technology.

For patients with balance disorders, we can use the accelerometers and gyroscopes in smartphones to remotely measure gait, evaluate eye movements that can help diagnose vestibular disorders, and use artificial intelligence algorithms to determine the most appropriate physicians for a patient's balance disorder. Smartphone-compatible peripherals can convert a phone into a medical device for use in a clinic (e.g., to record vestibular evoked myogenic potentials or audiometry).

Currently, smartphone-based health care is not intended to replace a visit to an audiologist, but rather to facilitate counseling, provide decision support, and collect data that is relevant to the hearing or balance condition.

In this issue of the Journal of the American Academy of Audiology, an article by Wu and colleagues titled "Comparison of In-Situ and Retrospective Self-Reports on Assessing Hearing Aid Outcomes" shows us how this technology is being leveraged to better understand and improve outcomes in hearing-impaired patients. An approach referred to as ecological momentary assessment (EMA) is a method of sampling data from a recent experience or in the current environment. Mobile technology lends itself to this application perfectly. $\mathrm{Wu}$ and his investigative team are on the forefront of describing the disassociation between laboratory-type outcome measures and measuring hearing outcomes in the real world. In-situ reports such as those provided by EMA offer a number of advantages over retrospective self-report measures.

I encourage you to read the interesting report in this issue by $\mathrm{Wu}$ and his team, as it provides us all with a look at the way technology can better assist us with managing our patients' care.
(C) 2021. American Academy of Audiology. All rights reserved. Thieme Medical Publishers, Inc., 333 Seventh Avenue, 18th Floor, New York, NY 10001, USA
DOI https://doi.org/ 10.1055/s-0041-1723790. ISSN 1050-0545. 
